\section{Adxanthromycin: A New Inhibitor of ICAM-1/LFA-1 Mediated Cell Adhesion from Streptomyces sp. NA-148}

Sir:

Intercellular adhesion molecule-1 (ICAM-1: CD54) and lymphocyte function-associated antigen-1 (LFA-1: CD11a/CD18) are cell surface adhesion molecules that interact with one another to promote a number of cellular interactions, including antigen-specific $\mathrm{T}$ lymphocyte stimulation $^{1,2)}$ and leukocyte adhesion to endothelium followed by emigration into sites of inflammation ${ }^{3,4)}$. In a number of in vivo studies, treatment of animals with monoclonal antibodies directed against either ICAM-1 or LFA-1 resulted in inhibition of the inflammatory process $^{5 \sim 9)}$. These results suggest that an inhibitor of ICAM-1 or LFA-1 could be a potential therapeutic drug for inflammatory and immunological diseases. Although the inhibition of ICAM-1/LFA-1 mediated adhesion of JY and HeLa cells by plant extracts has been reported $^{10,11)}$, there is no report on inhibitors from microbial metabolites. A search for inhibitors of the ICAM-1/LFA-1 system from microbial metabolites was carried out using two methodologies: a homotypic cell aggregation assay and a cell adhesion assay. During the screening program, we found a new inhibitor, designated adxanthromycin, in the cultured broth of strain Streptomyces sp. NA-148. In this communication we describe the screening, isolation, structure, and biological properties of adxanthromycin.

JY Epstein-Barr virus-transformed B lymphoblastoid cells were used for the homotypic cell aggregation assay ${ }^{12)}$. The assay was performed as the first screen for cell adhesion inhibitors. JY cells suspended in RPMI 1640 medium (Nikken Bio Medical Lab., Kyoto, Japan) were settled in flat-bottomed 96-well microtiter plates. The cells were stimulated with $50 \mathrm{ng} / \mathrm{ml}$ of phorbol-12myristate-13-acetate (PMA) with or without test samples, then incubated at $37^{\circ} \mathrm{C}$ for 24 hours. Cell aggregation was observed microscopically, and cytotoxicity of the samples was also determined simultaneously by a calorimetric analysis using WST-1 ${ }^{13}$. The culture without inhibitors showed large and compact aggregates, and the culture showed smaller clusters and nonaggregated cells in the presence of inhibitors. TS1/22
(anti-LFA-1 monoclonal antibody, ATCC, Manassasm VA) inhibited the formation of clusters or aggregates completely at $1 \mu \mathrm{g} / \mathrm{ml}$. The samples which inhibited the formation of cell aggregates without cytotoxicity were further tested in the adhesion assay using soluble ICAM-1 and LFA-1 positive SKW-3 T cell leukemia cells ${ }^{14)}$. SKW-3 cells (Hayashibara Biochemical Laboratories Inc., Fujisaki Cell Center, Okayama, Japan) were labeled with a fluorescence dye, BCECF-AM [2',7'-bis-(2-carboxylethyl)-5-(and-6)-carboxylfluorescein, acetoxymethyl ester $]^{15)}$ and then stimulated with $20 \mathrm{ng} / \mathrm{ml}$ of PMA at $37^{\circ} \mathrm{C}$ for 20 minutes. The cell suspension was added to a 96-well microtiter plate coated with immobilized soluble ICAM-1 and incubated in the presence of inhibitors at $37^{\circ} \mathrm{C}$ for 30 minutes. The adherent cells were solubilized with $0.1 \%$ Nonidet P-40 and fluorescence intensity was measured.

The producing strain identified as Streptomyces sp. NA-148 was found by screening a total of 2450 cultures of Actinomycetes. The strain was cultivated in a $100-\mathrm{ml}$ test tube containing $10 \mathrm{ml}$ of $\mathrm{TN}-2$ medium (glycerol $2.0 \%$, soybean powder $1.5 \%, \mathrm{CaCO}_{3} 0.3 \%, \mathrm{KH}_{2} \mathrm{PO}_{4}$ $0.01 \%, \mathrm{Na}_{2} \mathrm{HPO}_{4} \cdot 12 \mathrm{H}_{2} \mathrm{O} 0.04 \%, \mathrm{MgSO}_{4} \cdot 7 \mathrm{H}_{2} \mathrm{O} 0.05 \%$, $\mathrm{pH} 7.0$ ) at $27^{\circ} \mathrm{C}$ for 3 days on a rotary shaker $(230 \mathrm{rpm})$. The seed culture was inoculated into a 500-ml Erlenmeyer flask containing $100 \mathrm{ml}$ of the same medium, then cultured at $27^{\circ} \mathrm{C}$ for 5 days on a rotary shaker $(180 \mathrm{rpm})$. The whole culture was transferred into a 5-liter mini-jar fermentor containing 3 liters of the same medium and cultured at $27^{\circ} \mathrm{C}$ for 9 days (agitation; $250 \mathrm{rpm}$, aeration; 3 liters/minute). The fermentation broth (24 liters) was filtrated and the mycelial cake was extracted with acetone. After removal of the acetone, the aqueous solution was extracted with EtOAc. The extracts was subjected to silica gel chromatography with $\mathrm{CHCl}_{3}-\mathrm{MeOH}$ as the developing solvent to obtain a crude powder. The powder was purified by preparative HPLC (Senshu Pak ODS-H-5251) with MeOH-10 mm phosphate buffer, then washed with an acidic aqueous solution, isolating adxanthromycin (29 mg) as a pale yellow powder. The molecular formula, $\mathrm{C}_{42} \mathrm{H}_{40} \mathrm{O}_{17}\left[(\mathrm{M}-\mathrm{H})^{-}, m / z\right.$ 815.2252] was established by high-resolution FAB-MS measurement, which was supported by ${ }^{1} \mathrm{H}$ and ${ }^{13} \mathrm{C}$ NMR spectral data. The physico-chemical properties of adxanthromycin were as follows, mp. $233 \sim 235^{\circ} \mathrm{C}$ (dec.), $[\alpha]_{\mathrm{D}}+120.5^{\circ}$ (c 0.20, DMSO), UV $\lambda_{\max }$ in $\mathrm{MeOH}(\varepsilon)$ 
$272 \mathrm{~nm}(5,770), 315 \mathrm{~nm}(6,730)$ and $358 \mathrm{~nm}(4,390)$ and $\lambda_{\max }$ in $0.1 \mathrm{~N} \mathrm{NaOH}-\mathrm{MeOH}(\varepsilon) 254 \mathrm{~nm}(9,310), 315 \mathrm{~nm}$ $(6,400), 377 \mathrm{~nm}(8,170)$. The structure of adxanthromycin was deduced from NMR experiments including DQFCOSY, NOE difference, HMQC and HMBC. Adxanthromycin possesses a dimeric anthrone peroxide structure $^{16)}$ glycosidated with $\alpha$-galactose, as shown in Fig. 1. The ${ }^{13} \mathrm{C}$ NMR spectrum of adxanthromycin is shown in Fig. 2. The ${ }^{1} \mathrm{H}$ and ${ }^{13} \mathrm{C}$ NMR assignments (in DMSO$d_{6}+\mathrm{HCl}$ ) for adxanthromycin are indicated belows: $\mathrm{C}-1$ $\left(\delta_{\mathrm{C}} 138.7\right), \mathrm{C}-2\left(\delta_{\mathrm{C}} 129.1\right), \mathrm{C}-3\left(\delta_{\mathrm{C}} 155.8\right), \mathrm{C}-4\left(\delta_{\mathrm{C}} 110.7 / \delta_{\mathrm{H}}\right.$ $7.27), \mathrm{C}-4 \mathrm{a}\left(\delta_{\mathrm{C}} 148.9\right), \mathrm{C}-5\left(\delta_{\mathrm{C}} 117.0 / \delta_{\mathrm{H}} 6.29\right), \mathrm{C}-6\left(\delta_{\mathrm{C}}\right.$ $\left.136.3 / \delta_{\mathrm{H}} 7.07\right), \mathrm{C}-7\left(\delta_{\mathrm{C}} 114.4\right), \mathrm{C}-8\left(\delta_{\mathrm{C}} 159.6\right), \mathrm{C}-8 \mathrm{a}\left(\delta_{\mathrm{C}}\right.$ $124.9), \mathrm{C}-9\left(\delta_{\mathrm{C}} 189.6\right), \mathrm{C}-9 \mathrm{a}\left(\delta_{\mathrm{C}} 122.0\right), \mathrm{C}-10\left(\delta_{\mathrm{C}} 80.0\right)$, $\mathrm{C}-10 \mathrm{a}\left(\delta_{\mathrm{C}} 141.6\right), \mathrm{C}-11\left(\delta_{\mathrm{C}} 20.4 / \delta_{\mathrm{H}} 2.69\right), \mathrm{C}-12\left(\delta_{\mathrm{C}} 168.2\right)$,

Fig. 1. Structure of adxanthromycin.

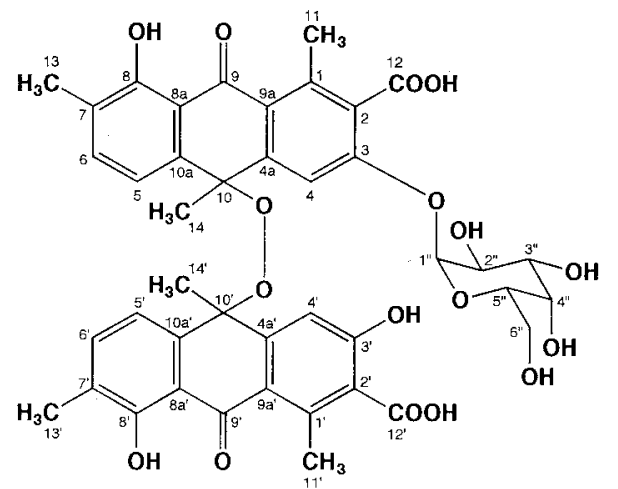

C-13 $\left(\delta_{\mathrm{C}} 15.3 / \delta_{\mathrm{H}} 2.19\right), \mathrm{C}-14\left(\delta_{\mathrm{C}} 33.2 / \delta_{\mathrm{H}} 1.39\right), \mathrm{C}-8-\mathrm{OH}$ $\left(\delta_{\mathrm{H}} 13.36\right), \mathrm{C}-1^{\prime}\left(\delta_{\mathrm{C}} 139.5\right), \mathrm{C}-2^{\prime}\left(\delta_{\mathrm{C}} 127.5\right), \mathrm{C}-3^{\prime}\left(\delta_{\mathrm{C}} 158.1\right)$, C-4 $\quad\left(\delta_{\mathrm{C}} 112.3 / \delta_{\mathrm{H}} 7.20\right), \quad \mathrm{C}-4 \mathrm{a}^{\prime}\left(\delta_{\mathrm{C}} 148.9\right), \quad \mathrm{C}-5^{\prime} \quad\left(\delta_{\mathrm{C}}\right.$ $\left.117.0 / \delta_{\mathrm{H}} 6.28\right), \mathrm{C}-6^{\prime}\left(\delta_{\mathrm{C}} 136.1 / \delta_{\mathrm{H}} 7.06\right), \mathrm{C}-7^{\prime}\left(\delta_{\mathrm{C}} 114.6\right)$, $\mathrm{C}-8^{\prime}\left(\delta_{\mathrm{C}} 159.6\right), \mathrm{C}-8 \mathrm{a}^{\prime}\left(\delta_{\mathrm{C}} 125.1\right), \mathrm{C}-9^{\prime}\left(\delta_{\mathrm{C}} 189.5\right), \mathrm{C}-9 \mathrm{a}^{\prime}$ $\left(\delta_{\mathrm{C}} 120.3\right), \mathrm{C}-10^{\prime}\left(\delta_{\mathrm{C}} 79.7\right), \mathrm{C}-10 \mathrm{a}^{\prime}\left(\delta_{\mathrm{C}} 141.2\right), \mathrm{C}-11^{\prime}\left(\delta_{\mathrm{C}}\right.$ $\left.20.7 / \delta_{\mathrm{H}} 2.68\right), \mathrm{C}-12^{\prime}\left(\delta_{\mathrm{C}} 168.9\right), \mathrm{C}-13^{\prime}\left(\delta_{\mathrm{C}} 15.3 / \delta_{\mathrm{H}} 2.19\right)$, $\mathrm{C}-14^{\prime}\left(\delta_{\mathrm{C}} 32.9 / \delta_{\mathrm{H}} 1.40\right), \mathrm{C}-8^{\prime}-\mathrm{OH}\left(\delta_{\mathrm{H}} 13.48\right), \mathrm{C}-1^{\prime \prime}\left(\delta_{\mathrm{C}}\right.$ $\left.96.8 / \delta_{\mathrm{H}} 5.73\right), \mathrm{C}-2^{\prime \prime}\left(\delta_{\mathrm{C}} 67.9 / \delta_{\mathrm{H}} 3.95\right), \mathrm{C}-3^{\prime \prime}\left(\delta_{\mathrm{C}} 69.3 / \delta_{\mathrm{H}}\right.$ $3.74), \mathrm{C}-4^{\prime \prime}\left(\delta_{\mathrm{C}} 68.2 / \delta_{\mathrm{H}} 3.75\right), \mathrm{C}-5^{\prime \prime}\left(\delta_{\mathrm{C}} 72.5 / \delta_{\mathrm{H}} 3.55\right), \mathrm{C}-6^{\prime \prime}$ $\left(\delta_{\mathrm{C}} 59.8 / \delta_{\mathrm{H}} 3.24,3.45\right)$.

Adxanthromycin inhibited the formation of the JY cell agrregates from $1.5 \mu \mathrm{g} / \mathrm{ml}$ in a dose dependent manner. The toxicity $\left(\mathrm{IC}_{50}\right)$ of adxanthromycin against JY cell was $15.2 \mu \mathrm{g} / \mathrm{ml}$. Adxanthromycin inhibited SKW-3 adhesion to soluble ICAM-1 in a dose-dependent manner with an $\mathrm{IC}_{50}$ of $18.8 \mu \mathrm{g} / \mathrm{ml}$. The cell toxicity $\left(\mathrm{IC}_{50}\right)$ of adxanthromycin against $\mathrm{SKW}-3$ was 110.0 $\mu \mathrm{g} / \mathrm{ml}$. The inhibitory activity of the compound in the cell-free receptor binding assay was examined in the manner reported previously ${ }^{17)}$. Adxanthromycin showed weak inhibition in the assay with an $\mathrm{IC}_{50}$ of $760 \mu \mathrm{g} / \mathrm{ml}$ in the cell-free system. Details of the screening method, biological activity and structure determination of adxanthromycin will be reported later.

Fig. $2 .{ }^{13} \mathrm{C}-\mathrm{NMR}$ spectrum of adxanthromycin $\left(125 \mathrm{MHz}\right.$, in DMSO- $\left.d_{6}+\mathrm{HCl}\right)$.

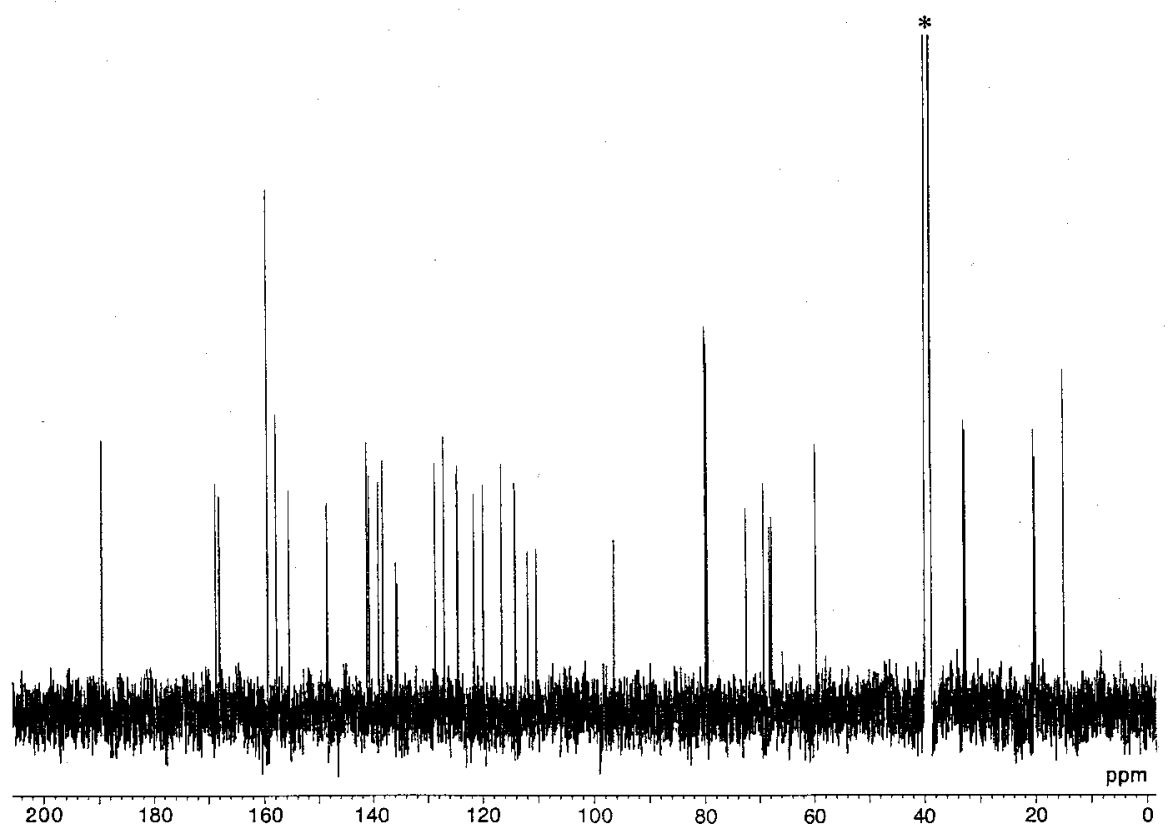


TSUKASA KoIWA

TAKAYUKi NAKano SENJI TAKAHASHI

HiroYUKI KoSHINO ${ }^{\dagger}$

Mika YamaZaKi ${ }^{\dagger \dagger}$

TOHRU TAKASHI ${ }^{\dagger \dagger}$

AKIRA NAKAGAWA*

Department of Biosciences, Teikyo University, 1-1 Toyosatodai, Utsunomiya 320-8551, Japan

† The Institute of Physical and Chemical Research (RIKEN), Hirosawa 2-1, Wako, Saitama 351-0198, Japan

${ }^{\dagger \dagger}$ Tokyo R \& D Center, Daiichi Pharmaceutical Co., Ltd., 1-16-13 Kita-kasai, Edogawa-ku, Tokyo 134-8630, Japan

(Received October 8, 1998)

\section{References}

1) Green, J. M.; X. G. Zheng, Y. Simizu, C. B. Thompson \& L. A. TurKa: T cell receptor stimulation, but not CD28 costimulation, is dependent on LFA-1-mediated events. Eur. J. Immunology 24: 265 272, 1994

2) Labuda, T.; J. Wendt, G. Hedlund \& M. Dohlsten: ICAM-1 costimulation induces IL-2 but inhibits IL-10 production in superantigen-activated human $\mathrm{CD}^{+} \mathrm{T}$ cells. Immunology 94: 496 502, 1998

3) Springer, T. A.: Adhesion receptors of the immune system. Nature 346: 425 434, 1990

4) Iigo, Y.; M. Suematsu, T. Higashida, J. Oheda, K. Matsumoto, Y. WaKabayashi, $\mathrm{Y}$. Ishimura, M. MiYasaka \& T. TAKashi: Constitutive expression of ICAM-1 in rat micrevascular systems analyzed by laser confocal microscopy. American J. Physiology 273: $138 \sim 147,1997$

5) Iigo, Y.; T. Takashi, T. Tamatani, M. Miyasaka, T. Higashida, H. Yagita, K. Okumura \& W. Tsukada: ICAM-1-dependent pathway is critically involved in the pathogenesis of adjuvant arthritis in rats. J. Immunology 147: $4167 \sim 4171,1991$

6) Nishikawa, K.; Y. Guo, M. Miyasaka, T. Tamatani, A. B. Collins, M. S. Sy, R. T. MCCluskey \& G. Andres: Antibodies to intercellular adhesion molecule $1 / \mathrm{lym}$ phocyte function-associated antigen 1 prevent crescent formation in rat autoimmune glomerulonephritis. $\mathbf{J}$. Experimental Medicine 177: 667 677, 1993

7) Kobayashi, Y.; K. Kawai, H. Honda, S. Tomida, N. Nimi, T. Tamatani, M. Miyasaka \& Y. Yoshikai: Antibodies against leukocyte function-associated antigen1 and against intercellular adhesion molecule- 1 together suppress the progression of experimental allergic encephalomyelitis. Cellular Immunology 164: 295 305, 1995

8) Nagase, T.; Y. Fukuchi, Y. Matsuse, T. Sudo, H. MATSUI \& H. ORIMO: Antagonism of ICAM-1 attenuates airway and tissue response to antigen in sensitized rats. Am. J. Respir. Crit. Care. Med. 151: 1244 1249, 1995

9) Bullard, D. C.; L. A. Hurley, I. Lorenzo, L. M. Sly, A. L. Beaudet \& N. D. Staite: Reduced susceptibility to collagen-induced arthritis in mice deficient in intercellular adhesion molecule-1. J. Immunology 157: $3153 \sim 3158,1996$

10) Musza, L. L.; L. M. Killar, P. Speight, S. McEllininey, C. J. Barrow, A. M. Gillum \& R. Cooper: Potent new cell adhesion inhibitory compounds from the root of Trichilia rubra. Tetrahedron 50: $11369 \sim 11378,1994$

11) Musza, L. L.; P. Speight, S. McElhiney, C. J. Barrow, A. M. Gillum; R. Cooper \& L. M. Killar: Cucurbitacins, cell adhesion inhibitors from Conobea scoparioides. J. Nat. Prod. 57: 1498 1502, 1994

12) Tominaga, T.; K. Sugie, M. Hirata, N. MoriI, J. Fukuda, A. Uchida, H. Imura \& S. Narumiya: Inhibition of PMA-induced, LFA-1-dependent lymphocyte aggregation by ADP ribosylation of the small molecular weight GTP binding protein, rho. J. Cell Biol. 120: $1529 \sim 1537,1993$

13) Ishikawa, M.; M. Shiga, K. Sasamoto, M. Mizoguchi \& P. G. HE: A new sulfonated tetrazolium salt that produces a highly water-soluble formazone dye. Chem. Pharm. Bull. 41: $1118 \sim 1122,1993$

14) Meyer, D. M.; M. L. Dustin \& C. P. Carron: Characterization of intercellular adhesion molecule-1 ectodomain (sICAM-1) as an inhibitor of lymphocyte function- associated molecule-1 interaction with ICAM-1. J. Immunology 155: $3578 \sim 3584,1995$

15) Gimbrone, M. A. Jr.; M. S. Obin, A. F. Brock, E. A. Luis, P. E. Hass \& C. A. Hebert, Y. K. Yip, D. W. Leung, D. G. Lowe, W. J. Kohr, W. C. Darbonne, K. B. BECHTOL \& J. B. BAKER: Endothelial interleukin-8: A novel inhibitor of leukocyte-endothelial interactions. Science 246: $1601 \sim 1603,1989$

16) Patel, M.; A. C. Horan, V. P. Gullo, D. Loebenberg, J. A. Marques, G. H. Miller \& J. A. Waitz: Oxanthromicin, a novel antibiotic from Actinomadura. J. Antibiotics 37: 413 415, 1984

17) Tominaga, Y.; Y. Kita, K. UchiYama, K. Sato, K. Sato, T. TAKASHI \& T. HoRIUCHI: Expression of a soluble form of LFA-1 and demonstration of its binding acitvity with ICAM-1. J. Immunol. Methods 212: $61 \sim 68,1998$ 